Trauma Berufskrankh 2009 · 11[Suppl 1]:55-59 DOI 10.1007/s10039-008-1425-6

Online publiziert: 13. September 2008

c) Springer Medizin Verlag 2008

H. Madry

Labor für Experimentelle Orthopädie, Klinik für Orthopädie

und orthopädische Chirurgie, Universitätsklinkum des Saarlandes, Homburg/Saar

\title{
Molekulare Therapie von traumatischen Knorpeldefekten
}

ner chondralen Verletzung jedoch sehr begrenzt. Bei der Gelenkknorpelreparatur entsteht ein Gewebe, das strukturelle Ähnlichkeiten mit hyalinem Gelenkknorpel hat. Demgegenüber wird Gelenkknorpelregeneration als die Wiederherstellung des Knorpels im Bereich des Defekts definiert, welcher dann nicht mehr vom benachbarten, unbeschädigten Gelenkknorpel zu unterscheiden ist. Knorpelregeneration tritt jedoch bei Erwachsenen für beide Arten von Defekten nicht spontan auf. Außerdem konnten weder mit konservativen noch mit operativen Behandlungsverfahren komplette und lang andauernde Knorpelregenerationen erreicht werden.

Orthopädische Chirurgen und Unfallchirurgen verwenden verschiedene Techniken, um Knorpeldefekte zu behandeln. Diese reichen von der konservativen Behandlung mit einer kontinuierlichen passiven Bewegung des Gelenks [27] bis hin zu verschiedenen chirurgischen Eingriffen wie markraumeröffnende Verfahren (Pridie-Bohrung, Mikrofrakturierung), autologe osteochondrale Transplantate oder die Transplantation von Chondrozyten. Jedoch führen auch derart elaborierte Verfahren nicht vorhersagbar zu einem Reparaturgewebe, das in seiner Struktur identisch mit dem normalen Knorpel ist und den physiologischen mechanischen Belastungen standhält [5]. Die Regeneration des hyalinen Gelenkknorpels bleibt daher weiterhin eine der größten Herausforderungen für Gelenkchirurgen $[5,11,28]$.

\section{Wachstumsfaktoren als therapeutische Kandidaten}

Da die Knorpelreparatur Ähnlichkeiten mit der embryonalen Chondrogenese hat, werden Strategien zur Steigerung der Chondrogeneseaktivität angestrebt. Wichtige Eigenschaften sind:

- die Zellularität des Reparaturgewebes,

- die Differenzierung der mesenchymalen Zellen in Chondrozyten sowie

- die Produktion und Aufrechterhaltung der Knorpelmatrix, die reich an Typ-II-Kollagen und Proteoglykanen ist.

Idealerweise sollten die therapeutischen Ansätze imstande sein, die Chondrogenese zu induzieren und die Zellproliferation mit Reifung und Matrixsynthese anzuregen. Wachstumsfaktoren wurden bereits vor einiger Zeit als wichtige Anwärter zur Unterstützung dieser Mechanismen erkannt [32]. Sie agieren über ihre spezifischen membrangebundenen Rezeptoren und wirken aufeinander ein, um ihre jeweiligen Tätigkeiten zu modulieren. Da es sich bei ihnen meist um Polypeptide handelt, vermindert ihre kurze pharmakologische Halbwertszeit ihre therapeutische Wirksamkeit [26]. So hat FGF2 (,fibroblast growth factor-2", Fibroblastenwachstumsfaktor 2) eine Plasmahalbwertszeit von weniger als $1 \mathrm{~h}$ und ist bereits einige Minuten nach seiner intraartikulären Injektion abgebaut [30]. Wachs-

Unterstützt durch die Deutsche Forschungsgemeinschaft und die Deutsche Arthrose-Hilfe. der fehlenden Blutgefäßversorgung ist der Zugang zu Vorläuferzellen im Bereich ei- 
a

b

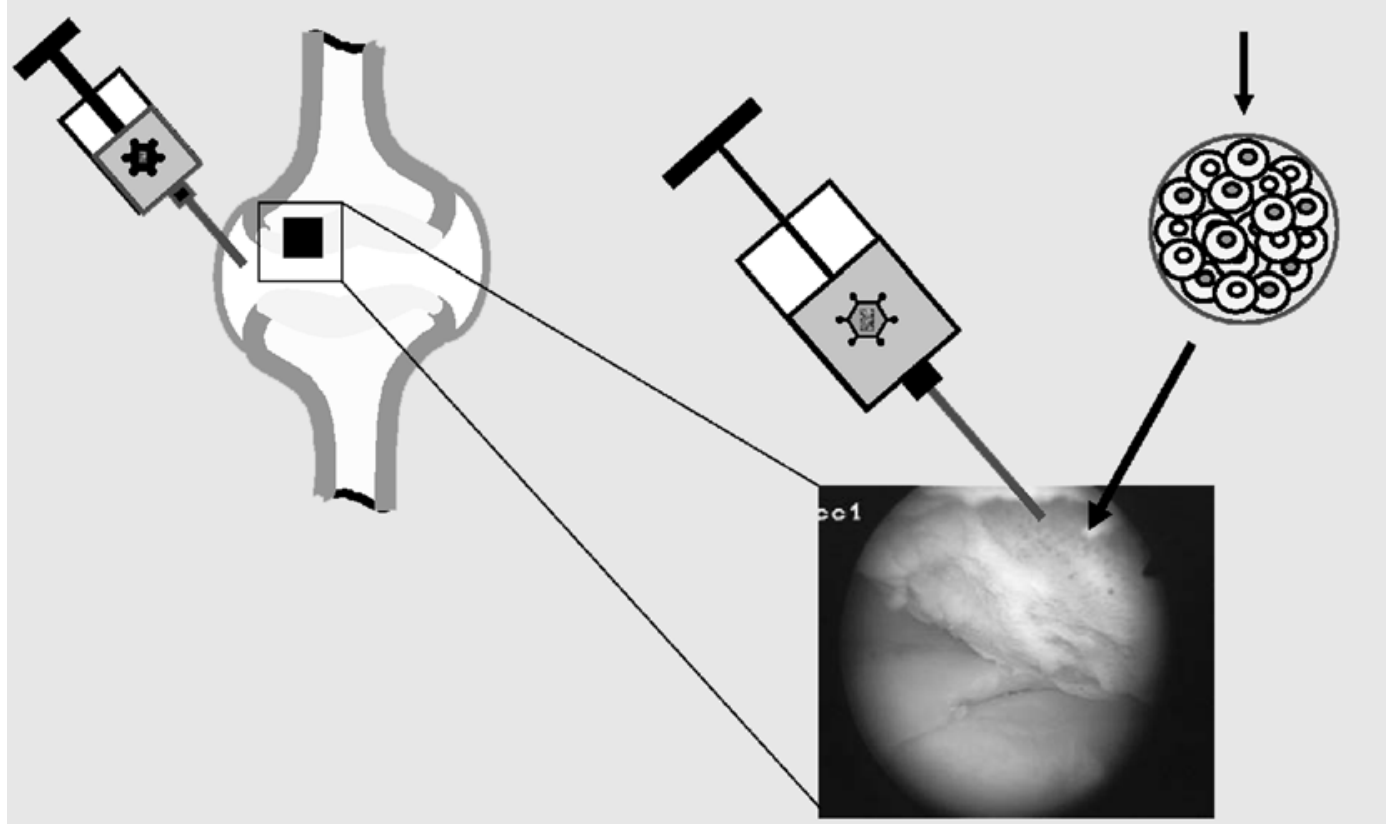

c

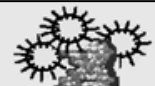

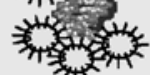

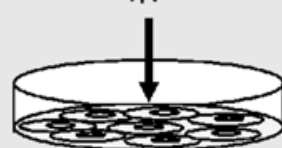

Abb. 14 Transfer therapeutischer Gene in umschriebene Gelenkknorpeldefekte, a intraartikuläre Injektion eines Genvehikels (ohne Arthrotomie), b Arthrotomie und direkte Verabreichung der Genvektoren in den Gelenkknorpeldefekt, c Arthrotomie und Transplantation von ex vivo genetisch modifizierten Zellen in den Defekt

tumsfaktoren zur Förderung der Chondrogenese sind:

- Mitglieder der TGF- $\beta$-Gruppe

(„transforming growth factor $\beta$ “), wie BMP-2 („bone morphogenetic protein 2") [29] oder BMP-7 [2],

- Mitglieder der Familie des Fibroblastenwachstumsfaktors wie FGF-2 [16] und

- der Wachstums- und Differenzierungsfaktor 5 („growth/differentiation factor 5“, GDF-5) [14].

DieZellproliferation wirdu. a. angeregtvon:

- FGF-2 [34] und

- insulinartigem Wachstumsfaktor I (,insulin-like growth factor I“, IGF-I) [31].

Besonders wirksame Kandidaten zur Anregung der Matrixsynthese sind

$$
\begin{aligned}
& \text { - IGF-I [33], } \\
& - \text { BMP-2 und } \\
& \text { - BMP-7. }
\end{aligned}
$$

Andere Strategien basieren auf Transkriptionsfaktoren, welche die Expression der
Gene direkt modulieren und in die Chondrogenese involviert sind, wie sox9 [4].

\section{Zielzellen für den Gentransfer in Gelenkknorpeldefekten}

Erfolgreiche Gentherapiestrategien für Knorpeldefekte hängen von der Effizienz der Bereitstellung therapeutischer Faktoren am beschädigten Knorpel ab. Im Allgemeinen ist es notwendig, therapeutische Dosen über einen ausgedehnten Zeitraum zu verabreichen, um andauernde chondrogene Antworten zu erzielen. sind:

Zielzellen für die Genübertragung

- Vorläuferzellen aus dem Knochenmark, die entweder spontan oder nach markraumeröffnenden Verfahren den Defekt füllen, sowie

- differenzierte Chondrozyten, die in den Defekt transplantiert werden.

Das größte Hindernis in der Entwicklung von effizienten Gentransferprotokollen bezüglich Gelenkknorpelschädigungen sind die bis heute eingeschränkte
Zugänglichkeit der Defekte und der Mangel an Genvehikeln, die zur Transduktion der Chondrozyten innerhalb ihrer Matrix fähig sind. Im letzten Jahrzehnt wurden bedeutende Fortschritte in einigen Bereichen erzielt. Die folgenden experimentellen Ansätze werden zurzeit eingesetzt, um Gene in Gelenkknorpeldefekte zu transferieren (- Abb. 1):

1. Injektion eines Genvehikels oder genetisch modifizierter Zellen in den Gelenkspalt ohne Arthrotomie

2. Arthrotomie mit Transplantation von ex vivo genetisch modifizierten Zellen in die fokalen Gelenkknorpeldefekte

3. Arthrotomie mit direkter Verabreichung von Genvektoren in den fokalen Gelenkknorpeldefekt

\section{Injektion ohne Arthrotomie}

Die intraartikuläre Injektion von Genvektoren ist historisch gesehen die erste angewendete Methode, da sie ein technisch einfaches Verfahren darstellt. Diese Strategie ist jedoch nicht sehr präzise, denn der therapeutische Vektor gelangt oft gar nicht 
in die Nähe des fokalen Knorpelschadens, sondern wird meist durch Synovialzellen aufgenommen. Prinzipiell können nur Gene angewendet werden, die bei Überexpression keine schädliche Wirkung innerhalb des Gelenkspalts entfalten. Daher mag eine intraartikuläre Vektorinjektion eher bei der rheumatoiden Arthritis oder Arthrose indiziert sein.

\section{Arthrotomie mit Transplantation ex vivo modifizierter Zellen}

Mit der direkte Transplantation genetisch modifizierter Zellen ex vivo in einen lokalisierten Gelenkknorpeldefekt sollten therapeutische Gene präziser an den Ort einer Knorpelverletzung gebracht werden als mit der intraartikulären Injektion von Genvektoren möglich.

Kang et al. [17] transplantierten erstmals genetisch modifizierte Zellen in vivo in einen Gelenkknorpeldefekt. In ihrer Studie wurden Chondrozyten mit einem retroviralen Vektor transduziert und in Fibringel eingebettet [17].

In anderen Studien wurden nichtvirale $[21,35]$, adenovirale $[3,15,25]$, retrovirale $[1,13,17,23,24]$ und rekombinante adeno-assoziierte virale (rAAV) Vektoren [19] verwendet. Obwohl hauptsächlich Chondrozyten transplantiert werden $[3,12,15,17,21,24,35]$, verwendeten einige Forscher auch autologe Knochenmarkaspirate [25] sowie perichondrale, periostale Zellen [10] und Muskelzellen.

Chondrozyten, transfiziert mit einem IGF-I exprimierenden Plasmidvektor, wurden in Alginatsphären verkapselt und in osteochondrale Knorpeldefekte transplantiert [22]. Bei beiden Gruppen, 3 und 14 Wochen postoperativ, waren die gesamte Gelenkknorpelreparatur und die Bildung des neuen subchondralen Knochens gegenüber Kontrollen erhöht [22].

Die Effekte einer FGF-2-Überexpression wurden in einem ähnlichen Modell studiert. Bei transfizierten Chondrozyten in Alginatsphäroiden [18] verbesserte FGF-2 die Zellmorphologie und die Architektur des neuen Gewebes und führte zu einer erheblichen Zunahme der gesamten Gelenkknorpelreparatur nach 3 und 14 Wochen in vivo. Nach 14 Wochen erhielt man einen niedrigen Punktwert für die Bewertung der Oberfläche des neuen
Trauma Berufskrankh 2009 · 11[Suppl 1]:55-59 DOI 10.1007/s10039-008-1425-6

(c) Springer Medizin Verlag 2008

\section{H. Madry \\ Molekulare Therapie von traumatischen Knorpeldefekten}

\section{Zusammenfassung}

Traumatische Defekte des hyalinen Gelenkknorpels sind ein ungelöstes Problem der Orthopädie und Unfallchirurgie. Keine der derzeit angewendeten Behandlungen resultiert in einer dauerhaften Knorpelregeneration. Obwohl das Konzept einer Gentherapie für Knorpelschäden zunächst elegant erscheint, müssen die verschiedenen Gentransfertechniken an das Problem eines umschriebenen Knorpeldefekts angepasst werden. Gegenwärtige Strategien basieren auf der Modulierung von Signalwegen der Knorpelheilung. Sie beinhalten die Stimulation der Zellproliferation und Matrixsynthese über einen di-

rekten Transfer oder durch Zelltransplantation. Vor allem Polypeptidwachstumsfaktoren verbessern die strukturelle Qualität des Reparaturgewebes. Erst ein besseres Verständnis der grundlegenden Aspekte der Knorpeldefektreparatur zusammen mit der Identifizierung von zusätzlichen molekularen Zielen können eine klinische Anwendung der Gentherapie für Knorpeldefekte erlauben.

\section{Schlüsselwörter}

Hyaliner Gelenkknorpel - Traumatischer Defekt · Dauerhafte Knorpelregeneration .

Gentherapie · Signalwege der Knorpelheilung

\section{Molecular therapy of traumatic cartilage defects}

\section{Abstract}

Traumatic defects of the hyaline articular cartilage are an unsolved problem in orthopedics and trauma surgery. None of the therapies currently used result in long-term cartilage regeneration. Although the concept of gene therapy for cartilage damage may appear attractive, the various gene transfer techniques need to be adapted to the treatment of cartilage defects. Strategies are currently based on the modulation of signaling pathways in cartilage healing. These include stimulating cell proliferation and matrix synthesis via direct transfer or cell transplanta- tion. In particular, polypeptide growth factors improve the structural quality of the repair tissue. $A$ better understanding of the basic aspects of cartilage defect repair, together with the identification of additional molecular targets are needed in order for gene therapy to be applied in a clinical setting.

\section{Keywords}

Hyaline articular cartilage - Traumatic defect · Long-term cartilage regeneration . Gene therapy · Signaling pathways of cartilage healing 
Gewebes und der Tidemark, was Hinweise auf den beginnenden Abbau des Reparaturgewebes in vivo gibt. Chondrozyten, die mit rAAV transduziert wurden und FGF-2 überexprimierten, zeigten eine ähnliche Verbesserung der Knorpelreparatur nach 12 Wochen [36].

Positive Effekte durch BMP-2 und IGF-I wurden erzielt, als perichondrale Zellen mit adenoviralen Vektoren transduziert und in chondrale Läsionen von Ratten appliziert wurden [9]. Defekte, die BMP-2 überexprimierende Zellen erhalten hatten, wiesen im Vergleich zu Zellen, denen IGF-I beigegeben worden war, eine höhere Zelldichte und einen höheren Proteoglykangehalt auf. Allerdings wurden nach Behandlung mit BMP-2 auch eine Osteophytenbildung und übermäßiges Wachstum des neu gebildeten Gelenkknorpels beobachtet. IGF-I-Überexpression führte zu keiner nachteiligen Folgeerscheinung [9].

Der nächste Schritt war, die soeben beschriebenen Strategien in einem großen Tiermodell in einem klinisch relevanten Rahmen zu prüfen. Hidaka et al. [12] setzten erstmals Pferde für solche ex vivo Projekte ein. Allogene Chondrozyten, transduziert mit einem adenoviralen Vektor, der BMP-7 beinhaltet, wurden durch Arthroskopie in Gelenkknorpeldefekte implantiert. Biopsien, die nach 4 Wochen entnommen wurden, zeigten ein verbessertes Erscheinungsbild des Knorpels in den Defekten, die mit BMP-7 überexprimierenden Zellen behandelt worden waren. Nach 8 Monaten gab es jedoch keine strukturellen Unterschiede zwischen den Gruppen. Dies war die erste Studie, in der eine biomechanische Prüfung des Reparaturgewebes durchgeführt wurde, obwohl keine funktionellen Unterschiede zwischen den Gruppen ermittelt werden konnten. Solch eine Funktionsauswertung ist unerlässlich, da alle Knorpelreparaturverfahren an der Verbesserung der mechanischen Eigenschaften des Reparaturgewebes gemessen werden müssen.

\section{Arthrotomie mit direkter Verabreichung von Genvektoren}

Der direkte Transfer der Kandidatengene in den Gelenkknorpel blieb lange Zeit ein Problem, da Gentransfertechniken, die auf nichtviralen, adenoviralen und retroviralen Vektoren basieren, keine Transgenexpression im Gelenkknorpel erlauben. Seit kurzer Zeit werden rAAV-Vektoren erfolgreich eingesetzt, um Gene durch direkte Vektorverabreichung in die Gelenkknorpeldefekte einzubringen [7, 2o]. Durch Applikation von rAAV-Vektoren mit einer FGF-2-Gensequenz direkt in Gelenkknorpeldefekte zeigte sich 4 Monate später die Knorpelreparatur signifikant verbessert [7].

\section{Zusammenfassung und Ausblick}

Die Regeneration des ursprünglichen hyalinen Knorpels bleibt die größte Herausforderung in der Gelenkchirurgie. Um dieses Ziel durch genbasierte Methoden zu erreichen, ist es entscheidend, die Inund Ex-vivo-Verfahren für den Gentransfer in Gelenkknorpeldefekte weiter zu verbessern.

Das Hauptproblem zurzeit ist die relativ kurze Expressionsdauer der Gene. Die Generierung von neuen Vektorkonstrukten, die zellspezifische oder regulierende Promotoren tragen, kann zur Verbesserung dieser Systeme beitragen. Zahlreiche Studien belegten, dass die Hauptaufgabe der transplantierten genetisch modifizierten Chondrozyten in der Produktion des therapeutischen Faktors liegt und nicht in der Repopulation des Defekts $[12,18,22,24]$. Es stellt sich die Frage, welche Menge an therapeutischem Chondrozytenprodukt gebildet werden muss, um den Knorpel optimal zu regenerieren. Anstatt eine große Menge genetisch modifizierter Zellen in den Defekt einzubringen, um ihn aufzufüllen, könnte es ausreichend sein, die Genproduktsynthese auf einen sehr spezifischen Platz und einen geeigneten Wirkspiegel zu beschränken. Der therapeutische Wirkstoff, der durch diese Zellen bereitgestellt wird, kann sowohl die Freigabe anderer Wachstumsoder Transkriptionsfaktoren als auch die Expression ihrer Rezeptoren beeinflussen und so auf parakrine Weise fungieren, um die Zellen anzuregen, die spontan den Defekt füllen, und sie in ihrer Chondrogenese zu stimulieren. Die Idee einer Transplantation genetisch modifizierten, durch Tissue Engineering veränderten Knorpels ist für die Reparatur von chondralen De- fekten besonders attraktiv, weil auf diese Weise ein vorgeformter Gewebeersatz gebildet wird, der einer erhöhten Chondrogenese unterworfen ist.

Die beachtliche technische Herausforderung bei diesen Ex-vivo-Strategien ist, die notwendigen Bestandteile (Zellen, Gene, bioresorbierbare Gerüstwerke, biologische Fixierung) in einen passenden Komplex zur Anwendung in einem klinischen Rahmen zu vereinen. Ebenso wichtig ist es, andere therapeutische Kandidaten zu prüfen, um den Prozess der Knorpelreparatur weiter verbessern $\mathrm{zu}$ können. Der entscheidende Beweis für die Anwendbarkeit dieser genbasierten Strategien zur Knorpelreparatur ist jedoch ihre Überprüfung in einem Großtiermodell, um einerseits eine klinisch relevante Situation zu erzeugen und andererseits diese Studien über einen hinreichend langen Beobachtungszeitraum durchzuführen [6]. Obwohl viel versprechende experimentelle Daten vorgelegt wurden, laufen derzeit keine Studien, in denen die Gentherapie fokaler Knorpeldefekte an Patienten angewendet wird.

Es ist eher unwahrscheinlich, dass die Gentherapie als einzelnes Verfahren die bestehenden klinischen Methoden für die Wiederherstellung der Gelenkknorpelbeschädigung ersetzen wird. Eher könnten genbasierte Therapien als Erweiterung für die zurzeit angewendeten Techniken dienen, z. B. bei markraumeröffnenden Verfahren, dem Ersatz eines gerissenen vorderen Kreuzbandes, und/oder einer Achskorrektur, welche die auf das Gelenk wirkenden Kräfte umverteilt.

\section{Korrespondenzadresse}

Prof. Dr. H. Madry

Labor für Experimentelle Orthopädie, Klinik für Orthopädie und orthopädische Chirurgie, Universitätsklinkum des Saarlandes, $66421 \mathrm{Homburg} / \mathrm{Saar}$ hmad@hotmail.com

Interessenkonflikt. Der korrespondierende Autor gibt an, dass kein Interessenkonflikt besteht.

\section{Literatur}

1. Adachi N, Sato K, Usas A et al. (2002) Muscle derived, cell based ex vivo gene therapy for treatment of full thickness articular cartilage defects. J Rheumatol 29: 1920-1930 
2. Asahina I, Sampath TK, Hauschka PV (1996) Human osteogenic protein-1 induces chondroblastic, osteoblastic, and/or adipocytic differentiation of clonal murine target cells. Exp Cell Res 222: 38-47

3. Baragi VM, Renkiewicz RR, Qiu L et al. (1997) Transplantation of adenovirally transduced allogeneic chondrocytes into articular cartilage defects in vivo. Osteoarthritis Cartilage 5: 275-282

4. Bi W, Deng JM, Zhang Z et al. (1999) Sox9 is required for cartilage formation. Nat Genet 22: 8589

5. Buckwalter JA, Mankin HJ (1998) Articular cartilage: degeneration and osteoarthritis, repair, regeneration, and transplantation. Instr Course Lect 47 487-504

6. Cucchiarini M, Madry H (2005) Gene therapy for cartilage defects. J Gene Med 7: 1495-1509

7. Cucchiarini M, Madry H, Ma C et al. (2005) Improved tissue repair in articular cartilage defects in vivo by rAAV-mediated overexpression of human fibroblast growth factor 2. Mol Ther 12: 229-238

8. Evans CH, Robbins PD, Ghivizzani SC et al. (1996) Clinical trial to assess the safety, feasibility, and efficacy of transferring a potentially anti-arthritic cytokine gene to human joints with rheumatoid arthritis. Hum Gene Ther 7: 1261-1280

9. Gelse K, Mark Klaus von der, Aigner T et al. (2003) Articular cartilage repair by gene therapy using growth factor-producing mesenchymal cells. Arthritis Rheum 48: 430-441

10. Gelse K, Mühle C; Franke O et al. (2008) Cell-based resurfacing of large cartilage defects: long-term evaluation of grafts from autologous transgeneactivated periosteal cells in a porcine model of osteoarthritis. Arthritis Rheum 58: 475-488

11. Gies T (1883) Ueber Heilung von Knorpelwunden. Dtsch Z Chir 18: 8-34

12. Hidaka C, Goodrich LR, Chen CT et al. (2003) Acceleration of cartilage repair by genetically modified chondrocytes over expressing bone morphogenetic protein-7. J Orthop Res 21: 573-583

13. Hirschmann F, Verhoeyen E, Wirth D et al. (2002) Vital marking of articular chondrocytes by retroviral infection using green fluorescence protein. Osteoarthritis Cartilage 10: 109-118

14. Hotten GC, Matsumoto T, Kimura M et al. (1996) Recombinant human growth/differentiation factor 5 stimulates mesenchyme aggregation and chondrogenesis responsible for the skeletal development of limbs. Growth Factors 13: 65-74

15. Ikeda T, Kubo T, Nakanishi T et al. (2000) Ex vivo gene delivery using an adenovirus vector in treatment for cartilage defects. J Rheumatol 27: 990996

16. Jentzsch KD, Wellmitz G, Heder G et al. (1980) A bovine brain fraction with fibroblast growth factor activity inducing articular cartilage regeneration in vivo. Acta Biol Med Ger 39: 967-971

17. Kang R, Marui T, Ghivizzani SC et al. (1997) Ex vivo gene transfer to chondrocytes in full-thickness articular cartilage defects: a feasibility study. Osteoarthritis Cartilage 5: 139-143

18. Kaul G, Cucchiarini M, Arntzen D et al. (2006) Local stimulation of articular cartilage repair by transplantation of encapsulated chondrocytes overexpressing human fibroblast growth factor 2 (FGF-2) in vivo. J Gene Med 8: 100-111

19. Kobayashi N, Koshino T, Uesugi M et al. (2002) Gene marking in adeno-associated virus vector infected periosteum derived cells for cartilage repair. J Rheumatol 29: 2176-2180
20. Madry H, Padera B, Seidel J et al. (2003) Efficient and persistent gene transfer into articular cartilage using recombinant adeno-associated virus vectors in vitro and in vivo. Hum Gene Ther 14: 393-402

21. Madry H, Cucchiarini M, Stein U et al. (2003) Sustained transgene expression in cartilage defects in vivo after transplantation of articular chondrocytes modified by lipid-mediated gene transfer in a gel suspension delivery system. J Gene Med 5: 502-509

22. Madry H, Kaul G, Cucchiarini M et al. (2005) Enhanced repair of articular cartilage defects in vivo by transplanted chondrocytes overexpressing insulin-like growth factor I (IGF-I). Gene Ther 12: 11711179

23. Mason JM, Grande DA, Barcia M et al. (1998) Expression of human bone morphogenic protein 7 in primary rabbit periosteal cells: potential utility in gene therapy for osteochondral repair. Gene Ther 5: 1098-1104

24. Mierisch CM, Wilson HA, Turner MA et al. (2003) Chondrocyte transplantation into articular cartilage defects with use of calcium alginate: the fate of the cells. J Bone Joint Surg Am 85-A: 1757-1767

25. Pascher A, Palmer GD, Steinert A, et al. (2004) Gene delivery to cartilage defects using coagulated bone marrow aspirate. Gene Ther 11: 133-141

26. Rogachefsky RA, Dean DD, Howell DS et al. (1993) Treatment of canine osteoarthritis with insulin-like growth factor-1 (IGF-1) and sodium pentosan polysulfate. Osteoarthritis Cartilage 1: 105-114

27. Salter RB (1989) The biologic concept of continuous passive motion of synovial joints. The first 18 years of basic research and its clinical application. Clin Orthop 242: 12-25

28. Seggel R (1904) Experimentelle Beiträge zur Anatomie und Pathologie des Gelenkknorpels. Studien über Knorpelwunden und Defekte. Dtsch Z Chir 21: 453-466

29. Sellers RS, Peluso D, Morris EA (1997) The effect of recombinant human bone morphogenetic protein-2 (rhBMP-2) on the healing of full-thickness defects of articular cartilage. J Bone Joint Surg Am 79: 1452-1463

30. Shida J, Jingushi S, Izumi T et al. (1996) Basic fibroblast growth factor stimulates articular cartilage enlargement in young rats in vivo. J Orthop Res 14: 265-272

31. Trippel SB (1995) Growth factor actions on articular cartilage. J Rheumatol Suppl 43: 129-132

32. Trippel SB (1997) Growth factors as therapeutic agents. Instr Course Lect 46: 473-476

33. Trippel SB, Van Wyk JJ, Foster MB et al. (1983) Characterization of a specific somatomedin-c receptor on isolated bovine growth plate chondrocytes. Endocrinology 112: 2128-2136

34. Trippel SB, Wroblewski J, Makower AM et al. (1993) Regulation of growth-plate chondrocytes by insulin-like growth-factor I and basic fibroblast growth factor. J Bone Joint Surg Am 75: 177-189

35. Ueblacker P, Wagner B, Krüger A et al. (2004) Inducible nonviral gene expression in the treatment of osteochondral defects. Osteoarthritis Cartilage 12: 711-719

36. Yokoo N, Saito T, Uesugi M et al. (2005) Repair of articular cartilage defect by autologous transplantation of basic fibroblast growth factor gene-transduced chondrocytes with adeno-associated virus vector. Arthritis Rheum 52: 164-170 